\title{
Existence and Permanence in a Diffusive KiSS Model with Robust Numerical Simulations
}

\author{
Kolade M. Owolabi and Kailash C. Patidar \\ Department of Mathematics and Applied Mathematics, University of the Western Cape, Private Bag X17, Bellville 7535, South Africa
}

Correspondence should be addressed to Kolade M. Owolabi; mkowolax@yahoo.com

Received 21 July 2015; Revised 27 November 2015; Accepted 1 December 2015

Academic Editor: Timothy R. Marchant

Copyright ( 2015 K. M. Owolabi and K. C. Patidar. This is an open access article distributed under the Creative Commons Attribution License, which permits unrestricted use, distribution, and reproduction in any medium, provided the original work is properly cited.

\begin{abstract}
We have given an extension to the study of Kierstead, Slobodkin, and Skellam (KiSS) model. We present the theoretical results based on the survival and permanence of the species. To guarantee the long-term existence and permanence, the patch size denoted as $L$ must be greater than the critical patch size $L_{c}$. It was also observed that the reaction-diffusion problem can be split into two parts: the linear and nonlinear terms. Hence, the use of two classical methods in space and time is permitted. We use spectral method in the area of mathematical community to remove the stiffness associated with the linear or diffusive terms. The resulting system is advanced with a modified exponential time-differencing method whose formulation was based on the fourth-order RungeKutta scheme. With high-order method, this extends the one-dimensional work and presents experiments for two-dimensional problem. The complexity of the dynamical model is discussed theoretically and graphically simulated to demonstrate and compare the behavior of the time-dependent density function.
\end{abstract}

\section{Introduction}

The study of reaction-diffusion problems has gained much attention over the years; such models are largely encountered in various fields and become increasingly useful tools for field engineers, mathematicians, conservation biologists, and population ecologists. In this paper, we describe the current state of the linear Kierstead-Slobodkin [1] and Skellam [2] problem (known as KiSS model) and give possible extension to such reaction-transport problem in a nonlinear form. The first theoretical approach to the KiSS model dates back to the early 1950s, studying the dynamic of reaction-diffusion problem for a population growing on a patch of finite size (say, $L$ ) that is surrounded by a deadly environment with infinite mortality [3].

Understanding the conditions that could guarantee the extinction and persistence of species populations in large but finite domains is another focus area of this paper. Further, determination of the critical patch size ensuring the sustenance of population is another vital area to consider, and the critical patch size depends on some factor, namely, the species population in the patch, geometrical patch, boundaries type, and the reproduction kinetics of species population. In what follows, we will present the extended nonlinear KiSS model and determine its critical patch size and reproduction processes with hostile boundaries.

\section{Mathematical Analysis of the Main Equation}

To start with, we let the critical patch size be $L$ and consider the reaction-diffusion problem

$$
\frac{\partial u}{\partial t}=\delta \frac{\partial^{2} u}{\partial x^{2}}+\tau \mathscr{F}(u)^{\rho}
$$

on the closed interval $[0, L]$ subject to homogeneous Dirichlet boundary and initial conditions

$$
\begin{aligned}
& u(0, t)=u(L, t)=0, \\
& u(x, 0)=u_{0}(x), \\
& \mathscr{F}^{\prime}(0)>0,
\end{aligned}
$$

where $u(x, t)$ is the species density, $\delta>0$ is the diffusion coefficient, $\tau$ is the growth rate, and $\rho>0$ is the critical 
exponent parameter that determines whether model (1) is linear $(\rho=1)$ or nonlinear $(\rho \geq 2)$. From the given boundary conditions, we observed that the critical patch size corresponds to the borderline between species population extinction and existence; we thus linearize (1) and (2) about $u \equiv 0$, the washout state. If $u=0$ is stable, we have a total extinction of the species population. But if $u=0$ is unstable (nontrivial case), we have a state that corresponds to the persistence or survival of the species. For simplicity, we relax the nonlinear condition and let $\rho=1$. We linearize (1) and (2) about $u(x)=0$ to obtain

$$
\begin{aligned}
& \frac{\partial u}{\partial t}=\delta \frac{\partial^{2} u}{\partial x^{2}}+\tau \mathscr{F}^{\prime}(0) u \\
& \quad u(0, t)=u(L, t)=0, u(x, 0)=u_{0}(x), \mathscr{F}^{\prime}(0)>0 .
\end{aligned}
$$

For linear dynamics, it is permitted to seek for solutions of the form

$$
u(x, t)=\exp \left(\tau \mathscr{F}^{\prime}(0) t\right) U(x, t) .
$$

By using this ansatz in (3), we obtain a simple diffusion equation:

$$
\begin{aligned}
& \frac{\partial U}{\partial t}=\delta \frac{\partial^{2} U}{\partial x^{2}} \\
& \qquad(0, t)=U(L, t)=0, U(x, 0)=U_{0}(x),
\end{aligned}
$$

and the solution of (3) is given by $[4,5]$

$$
\begin{aligned}
& u(x, t) \\
& \quad=\sum_{k=1}^{\infty} \omega_{k} \exp \left\{\left[\tau \mathscr{F}^{\prime}(0)-\delta\left(\frac{\pi k}{L}\right)^{2}\right] t\right\} \sin \left(\frac{\pi k x}{L}\right),
\end{aligned}
$$

where $\omega_{k}$ are the coefficients to be determined by the Fourier series expansion of $u_{0}(x)$. The unstable trivial solution $u(x) \equiv$ 0 could result in the growth and persistence of the species population if $\lambda_{k}=\tau \mathscr{F}^{\prime}(0)-\delta(\pi k / L)^{2}>0$ for some values of $k$. The growth rates $\lambda_{k}$ usually decreased monotonically with $k$, and $\lambda_{1}<0$. By setting the dominant mode $k=1$, we can determine the fate of species population $L<\pi \sqrt{\delta / \tau \mathscr{F}^{\prime}(0)}$ which guarantee the total washout of the species. The critical patch size known as the KiSS size is defined by the expression

$$
L_{c}=\pi \sqrt{\frac{\delta}{\tau \mathscr{F}^{\prime}(0)}} .
$$

If $L<L_{c}, u(x, t) \rightarrow 0$ as $t \rightarrow \infty$. The population is wiped out from its initial condition; no nontrivial steady state develops. Instability (bifurcation) could only arise if the patch size $L$ is increased. The state $u(x)=0$ loses its stability at the point $L_{c}$, and due to nonlinear effect $u$ begins to grow and saturates with time $t$. So as $u(x, t) \rightarrow \widehat{u}(x)$, a steady state (1) is determined by

$$
\delta \frac{\partial^{2} \widehat{u}}{\partial x^{2}}+\tau \mathscr{F}(\widehat{u})^{\rho}=0, \quad \widehat{u}(0)=\widehat{u}(L)=0, \rho=1 .
$$

It is obvious that, for any value of $L$, the trivial solution $\widehat{u}(x) \equiv 0$ holds for (8). The interest of the present work is in the solutions for which $\widehat{u}(x) \geq 0$. As a result of the spatial symmetry of (1) and (8), one expects the solution in $x$ to be symmetric about the midpoint $x=L / 2$, and it is reasonable to assume that the species density of nontrivial steady states could reach its maximum point, say $u_{\max }$ at the midpoint $d \widehat{u}(x) / d x(L / 2)=0$, since $\widehat{u}=0$ at the boundaries. Multiplying (8) with $d \widehat{u} / d x$ and integrating the result with respect to $x$ on $[0, L]$ yield

$$
\frac{\delta}{2}\left(\frac{d \widehat{u}}{d x}\right)^{2}+\tau \Psi(\widehat{u})=\tau \Psi\left(u_{\max }\right),
$$

where $\Psi(\widehat{u})=\int_{0}^{\widehat{u}} \mathscr{F}(s) d s$. If we simplify (9) by separating the variables and integrate it from 0 to $L / 2$ with respect to $x$, we obtain

$$
L=\sqrt{\frac{2 \delta}{\tau}} \int_{0}^{u_{\max }} \frac{d \widehat{u}}{\sqrt{\Psi\left(u_{\max }\right)-\Psi}(\widehat{u})} .
$$

We can rescale with $\mu=\widehat{u} / u_{\max }$ to obtain

$$
L=\sqrt{\frac{2 \delta}{\tau}} \int_{0}^{1} \frac{d \mu}{\sqrt{\Psi\left(u_{\max }\right)-\Psi}(\widehat{u})} .
$$

Equation (11) is usually called a time map, from where $u_{\max }$ can be determined implicitly as a function of $L$. We can also change the origin to $L / 2$; that is, by setting $x \rightarrow x-L / 2$, set $z=2 x / L-1$; we have

$$
\begin{aligned}
& \delta \frac{d^{2} \widehat{u}}{d z^{2}}+\lambda \mathscr{F}(\widehat{u})=0, \\
& \qquad \widehat{u}(0)=u_{\max }, \widehat{u}(1)=0,\left.\frac{d \widehat{u}}{d z}\right|_{z=0},
\end{aligned}
$$

where $\lambda=L^{2} \tau / 4 \delta$ is the eigenvalue. In the spirit of [4], we have

$$
L^{2} \approx \frac{\delta \pi^{3}}{4 \tau} u_{\max }\left[\int_{0}^{1} \frac{\mu \mathscr{F}\left(\mu u_{\max }\right) d \mu}{\sqrt{1-\mu^{2}}}\right]^{-1} .
$$

By taking the limit as $u_{\max } \rightarrow 0$, we obtain the critical patch size

$$
\begin{aligned}
L_{c}^{2} & \approx \lim _{u_{\max } \rightarrow 0} L^{2}=\frac{\delta \pi^{3}}{4 \tau \mathscr{F}^{\prime}(0)}\left[\int_{0}^{1} \frac{\mu^{2} d \mu}{\sqrt{1-\mu^{2}}}\right]^{-1} \\
& =\frac{\delta \pi^{2}}{\tau \mathscr{F}^{\prime}(0)}
\end{aligned}
$$

which is similar to (7). In conclusion, we can say that the nonuniform stationary solution of reaction-diffusion equation (1) exists if $L>L_{c}$ and that the trivial solution $\widehat{u} \equiv 0$ coexists. 
In other physical contexts, (1) is referred to as a blow-up problem [6-9]. It is a known theory from ODEs that solutions may not exist for $t \rightarrow \infty$ (global existence) but could tend to $\infty$ for a finite time $t$ (local existence). So, similar things happen to reaction-diffusion problems. On a large but finite interval, say $[-L, L]$, problem (1) can be written in integral form:

$$
u(t)=e^{t \Delta} u_{0}+\int_{0}^{t} e^{(t-s) \Delta} u(s)^{p} d s,
$$

where $u(t)=u(\cdot, t)$ and $\Delta=(d / d x)^{2}$ are subject to homogeneous Dirichlet boundary conditions on $[-L, L]$. The nonlinear term $\mathscr{F}(u)^{p}$ is locally Lipschitz on $C_{0}([-L, L])$, and continuous functions vanished at $\pm L$. By argument, if $u_{0}$ is nonnegative function in $C_{0}([-L, L])$, then there exists a nonnegative local solution $u(t)$ of (15). A reasonable question to ask is, does $u(x, t) \rightarrow \infty$ as $t \rightarrow T$ for all $x \in(-L, L)$ or singularity is restricted to a smaller interval? To answer this, we give the following theorem [10].

Theorem 1. Let $\mathscr{F}(u)=u^{p}$ and $u:[-L, L] \rightarrow[0, \infty)$ be the $C^{2}$ solution of the stationary problem

$$
0=\frac{d^{2} u}{d x^{2}}+u^{p}, \quad 0=u( \pm L), 0<u,-L<x<L .
$$

Let $\varphi=k u$, where $k>1$ is chosen so that the maximum solution $u(t)$ of (15) poses finite existence time, say T. If $p \geq 2$ and is sufficiently large, then $\lim _{t \rightarrow T} u(x, t)$ exist and are finite for all $x \neq 0$. In other words, singularity (discontinuity) occurs at the point $x=0$.

Proof. The proof of Theorem 1 can be found in [10].

\section{Formulation of Adaptive Methods in Space and Time}

Considerations have been given to the use of spectral methods as alternative to conventional finite differences [11], because they are capable of removing the stiffness property in the linear term of (1). Knowing that the reaction-diffusion problem can be split into the linear and nonlinear terms, the use of time and space methods is permitted [12-14]. Also, based on the known integrating factor technique, we are going to formulate the theory of spectral method in two spatial dimensions. By applying the integrating factor technique to Fourier transform systems (1), we have

$$
\begin{aligned}
U_{t}\left(\chi_{x}, \chi_{y}, t\right)= & -\left(\chi_{x}^{2}+\chi_{y}^{2}\right) U\left(\chi_{x}, \chi_{y}, t\right) \\
& +\mathscr{F}[f(u(x, y, t))],
\end{aligned}
$$

where $U$ is the double Fourier transforms of species density $u(x, y, t)$. In other words,

$$
\begin{aligned}
\mathscr{F}[u(x, y, t)] & =U\left(\chi_{x}, \chi_{y}, t\right) \\
& =\iint_{-\infty}^{\infty} u(x, y, t) e^{-i\left(\chi_{x} x+\chi_{y} y\right)} d x d y .
\end{aligned}
$$

To explicitly remove the adherent stiffness in the second partial derivatives, we let $\Omega^{2}=\left(\chi_{x}^{2}+\chi_{y}^{2}\right)$ and set $U=e^{-\Omega^{2} t} \bar{U}$, so that

$$
\partial_{t} \bar{U}=e^{\Omega^{2} t} \mathscr{F}[f(u, v, w)] .
$$

Next, we need to discretize the square domain by considering the equispaced points $N_{x}$ and $N_{y}$ in the spatial directions of $x$ and $y$. We employ the discrete fast Fourier transform (DFFT) $[12,13,15]$ to transform (19) to a system of ordinary differential equations (ODEs):

$$
\partial_{t} \bar{U}_{i, j}=e^{\Omega_{i, j}^{2} t} \mathscr{F}\left[f\left(u_{i, j}, v_{i, j}, w_{i, j}\right)\right],
$$

where $u_{i, j}=u\left(x_{i}, y_{j}\right), v_{i, j}=v\left(x_{i}, y_{j}\right), w_{i, j}=w\left(x_{i}, y_{j}\right)$, and $\Omega_{i, j}^{2}=\chi_{x}^{2}(i)+\chi_{y}^{2}(j)$. Boundary conditions are now set at extremes of the domain. Also in the experiment, we use a square Fourier node of $256 \times 256$ for $N_{x}=N_{y}=N=256$. At this stage, we have converted the system to ODEs; also, the stiffness has been removed. It should be mentioned that we can now use any explicit scheme to advance in time.

By adopting the notation of order $s$ Runge-Kutta (RK) methods, with time step $\Delta t$, we can advance from $t_{n}=n \Delta t$ to $t_{n+1}=(n+1) \Delta t$ for the ODE:

$$
u_{t}=f(u, t): u_{n+1}=\sum_{i=1}^{s} c_{i} k_{i}
$$

where

$$
k_{i}=\Delta t f\left(t_{n}+\alpha_{i} \Delta t, u_{n}+\sum_{j=1}^{i-1} \beta_{i j} k_{j}\right) .
$$

In the interest of the present paper, we apply the general explicit Runge-kutta scheme to (20) for $\bar{U}_{i j}$. For brevity, we denote $\mu_{i}$ to be $k^{\prime}$ in $\bar{U}_{i j}$ and set the replacement variable as

$$
\overline{\mu_{i}}=\mu_{i} \exp \left(-\Omega^{2} t_{n}\right) .
$$

Finally, the $s$-stage RK scheme becomes

$$
U_{n+1}=\exp \left(-\Omega^{2} \Delta t\right)\left[U_{n}+\sum_{i=1}^{s} c_{i} \overline{\mu_{i}}\right],
$$

with modified term

$$
\overline{\mu_{i}}=\exp \left(\Omega^{2} \alpha_{i} \Delta t\right) \Delta t \mathscr{F}\left[f\left(\mathscr{F}^{-1}\left(U_{n+\alpha_{i}}\right)\right)\right]
$$

and $U$ values at intermediate step

$$
U_{n+\alpha_{i}}=\exp \left(-\Omega^{2} \alpha_{i} \Delta t\right)\left[U_{n}+\sum_{j=1}^{i-1} \beta_{i j} \overline{\mu_{j}}\right] .
$$

The indication here is that one experiments entirely in the spectral domain and inverts transform to get $u$. It should be noted that, by removing the associated stiffness, one can 
accurately implement the resulting system of ODEs with any higher-order time stepping schemes; see [16-19] for details.

Next, we formulate the fourth-order exponential timedifferencing Runge-Kutta (ETDRK4) method by following closely most notations used by Cox and Matthews [16]. By multiplying through by the term $e^{-\mathbf{L} t}$, known as the integrating factor, we obtain (17) in the form

$$
e^{-\mathbf{L} t} U_{t}=e^{-\mathbf{L} t} \mathbf{L} u+e^{-\mathbf{L} t} \mathbf{N}(u, t)
$$

On integrating (27) over a single time step in the interval of length $h$, that is, $\left[t=t_{n}, t_{n+1}=t_{n}+h\right]$, we have

$$
\begin{aligned}
U\left(t_{n+1}\right)= & e^{\mathbf{L} h} U\left(t_{n}\right) \\
& +e^{\mathbf{L} h} \int_{0}^{h} e^{-\mathbf{L} \tau} \mathbf{N}\left(u\left(t_{n}+\tau\right), t_{n}+\tau\right) d \tau,
\end{aligned}
$$

where $\mathbf{L}$ is the linear operator and $\mathbf{N}$ is the Fourier transform of the nonlinear reaction functions. A direct application of the standard fourth-order Runge-Kutta method leads to a scheme by Cox and Matthew [16], known as the ETDRK4. Numerical experiments have shown that the cancellation errors in their scheme were too pronounced, which caused the method to suffer serious numerical instability and order reduction in its computation. We utilize in this work the modified Krogstad [18, 20] version of the ETDRK4 scheme that has been formulated to overcome the inherent numerical stability with smaller local truncation error,

$$
\begin{aligned}
U_{n+1} & \\
= & e^{\mathbf{L} h} U_{n} \\
& +h\left[4 \varphi_{3}(\mathbf{L} h)-3 \varphi_{2}(\mathbf{L} h)+\varphi_{1}(\mathbf{L} h)\right] \mathbf{N}\left(u_{n}, t_{n}\right) \\
& +2 h\left[\varphi_{2}(\mathbf{L} h)-2 \varphi_{3}(\mathbf{L} h)\right] \mathbf{N}\left(\mu_{2}, t_{n}+\frac{h}{2}\right) \\
& +2 h\left[\varphi_{2}(\mathbf{L} h)-2 \varphi_{3}(\mathbf{L} h)\right] \mathbf{N}\left(\mu_{3}, t_{n}+\frac{h}{2}\right) \\
& +h\left[\varphi_{3}(\mathbf{L} h)-2 \varphi_{2}(\mathbf{L} h)\right] \mathbf{N}\left(\mu_{4}, t_{n}+h\right),
\end{aligned}
$$

with the stages $\mu_{i}$ given as

$$
\begin{aligned}
\mu_{2}= & e^{\mathbf{L} h / 2} U_{n}+\left(\frac{\mathbf{L} h}{2}\right) \varphi_{1}\left(\frac{\mathbf{L} h}{2}\right) \mathbf{N}\left(u_{n}, t_{n}\right), \\
\mu_{3}= & e^{\mathbf{L} h / 2} U_{n} \\
& +\left(\frac{\mathbf{L} h}{2}\right)\left[\varphi_{1}\left(\frac{\mathbf{L} h}{2}\right)-2 \varphi_{2}\left(\frac{\mathbf{L} h}{2}\right)\right] \mathbf{N}\left(u_{n}, t_{n}\right) \\
& +h \varphi_{2}\left(\frac{\mathbf{L} h}{2}\right) \mathbf{N}\left(\mu_{2}, t_{n}+\frac{h}{2}\right), \\
\mu_{4}= & e^{\mathbf{L} h} U_{n}+h\left[\varphi_{1}(\mathbf{L} h)-2 \varphi_{2}(\mathbf{L} h)\right] \mathbf{N}\left(u_{n}, t_{n}\right) \\
& +2 h \varphi_{2}(\mathbf{L} h) \mathbf{N}\left(\mu_{3}, t_{n}+h\right),
\end{aligned}
$$

and with functions $\varphi_{1,2,3}$ defined as

$$
\begin{aligned}
\varphi_{1}(z) & =\frac{e^{z}-1}{z}, \\
\varphi_{2} & =\frac{e^{z}-1-z}{z^{2}}, \\
\varphi_{3} & =\frac{e^{z}-1-z-z^{2} / 2}{z^{3}}
\end{aligned}
$$

which precisely coincide with the terms in the Lie group methods by Munthe-Kaas [21].

\section{Numerical Experiments}

In this section, we intend to present the numerical results in one and two dimensions. We expect our results to reflect the mathematical results. We simulate the KiSS model in one and two dimensions with the ETDRK4 method presented in Section 3. Its stability analysis and comparison with other existing higher-order schemes when applied to some reaction-diffusion problems can be found in [18-20,22] and references therein.

4.1. One-Dimensional Example. In one dimension, consider the nonlinear KiSS model

$$
u_{t}=\delta u_{x x}+\tau u^{p}
$$

subject to the boundary conditions $u(0, t)=u(L, t)=0$ and initial population

$$
u(x, 0)=\frac{1}{2 \cosh \omega x}
$$

which has exponential decay $e^{-\omega|x|}$ as $x \rightarrow \infty$. Mathematically, one expects traveling waves to arise from such initial condition on an infinite domain $(-\infty, \infty)$ which we have truncated in the interest of the work here at some large but finite value, $L>0$. For the extended nonlinear example, we choose $p=2$. In Figure 1, we illustrate the permanence effect and stability of the existence state via numerical simulations of (32). Ecological parameters $\omega=0.25, \delta=0.5$, and $p=2$ are chosen to ensure the long-term survival of the species population. It is noticeable that the ETDRK4 scheme converged even at the expense of variability of the growth rates $\tau=1 / 2,1 / 4,1 / 8$, and $1 / 16$ for panels (a-d), respectively. It should be noted that the numerical simulation depicts the convergence of the solution to a positive survival state with population density of 1 , for all $t>0$ and $L \in[0,1]$.

In the second experiment, we utilize a random initial condition which we computed as

$$
\mathrm{u}=\operatorname{zeros}(\mathrm{N}, 1)+.02 * \operatorname{rand}(\mathrm{N}, 1),
$$

to permit the use of large domain size: $L=200$.

In Figure 2, we demonstrate the effect of diffusion on model (32) by allowing the species population to evolve from the random initial condition; parameters are fixed as $\tau=$ 2.5, $p=2$, and $t=1200$; others are given in the figure 


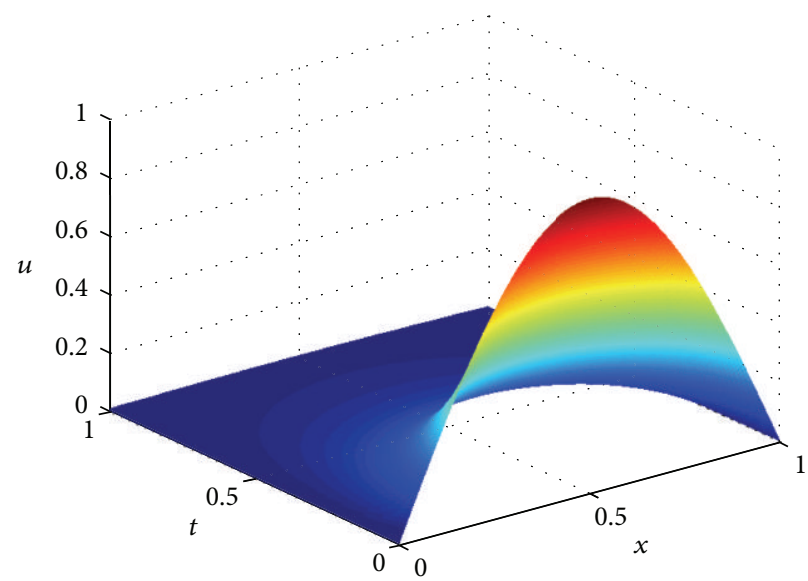

(a)

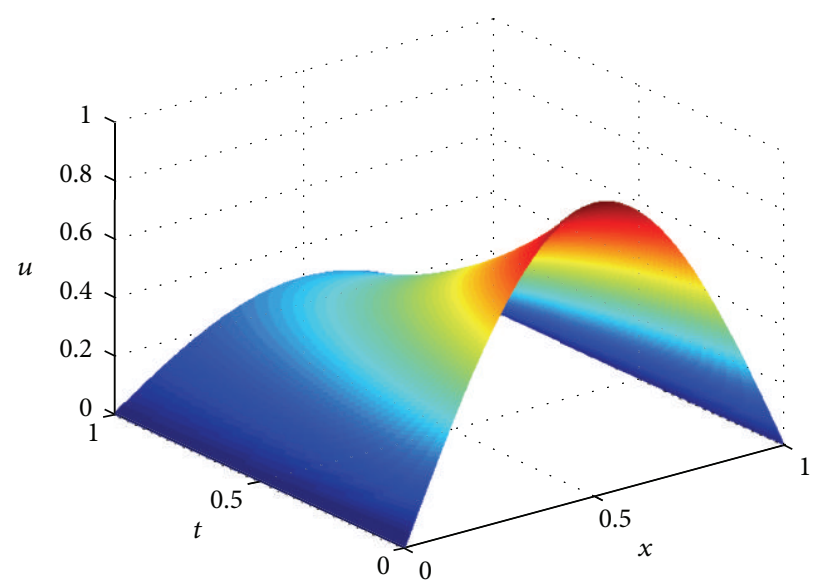

(c)

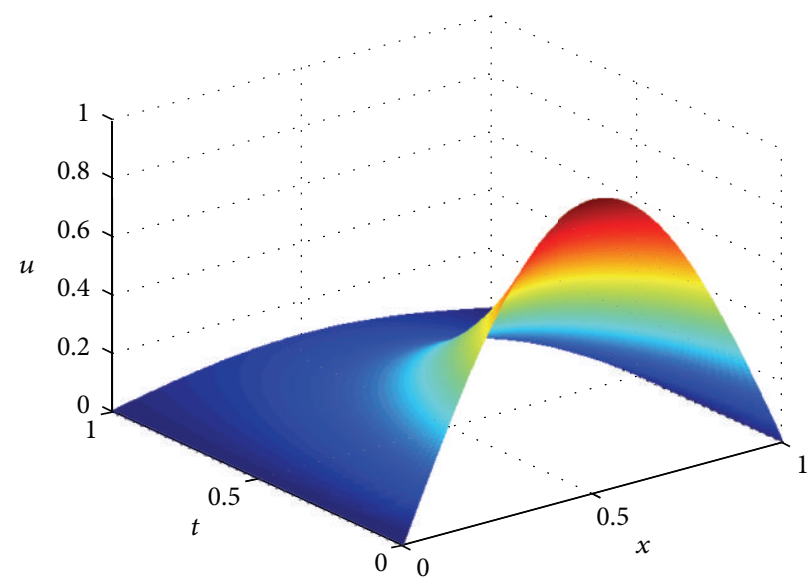

(b)

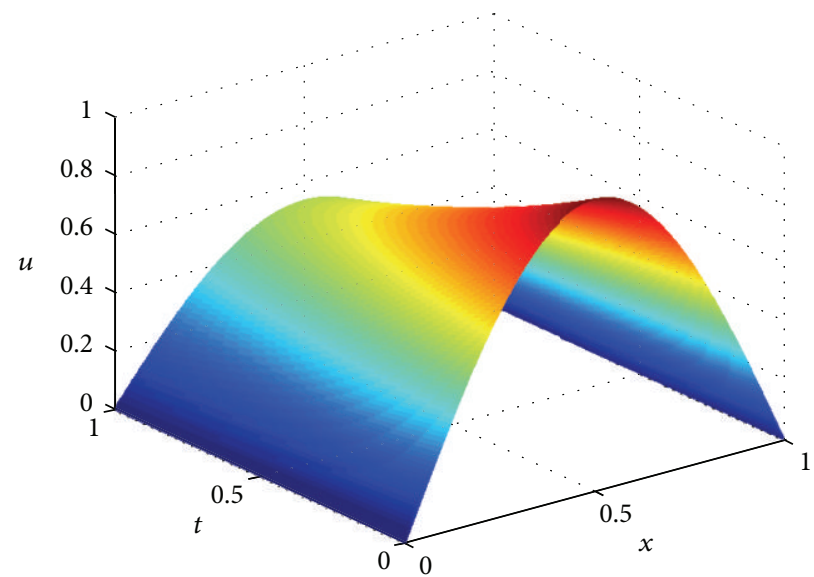

(d)

FIgURE 1: Permanence and existence of one-dimensional model (32) with Dirichlet boundary conditions at $t=1$.

caption. In the experiment, as the value of $\delta$ is decreasing, the species population oscillate in phase, but a stable steadystate solution is obtained for $\delta>1$. We should also mention that if $p \gg 2$ as $t \rightarrow \infty$, blow-up phenomena occur which correspond to the total extinction of the species.

4.2. Two-Dimensional Example. Bear in mind that it is in higher dimensions that the mathematical ideas reported in this paper become of serious value. Hence, we give an extension to the numerical experiments in two-dimensional space. Also, we experiment with two different initial conditions to study the behavior of the problem.

In two dimensions, we consider the reaction-diffusion problem

$$
\begin{aligned}
& u_{t}=\delta\left(u_{x x}+u_{y y}\right)+\tau u^{p}, \\
& \qquad(x, y) \in[-L, L] \times[-L, L] .
\end{aligned}
$$

We choose to illustrate the numerical algorithms with choice of initial condition:

$$
\begin{aligned}
u(x, y, 0)= & 10 \exp \left[-8\left((x+1)^{2}+(y+1)^{2}\right)\right] \\
& +10 \exp \left[-8\left((x-1)^{2}+(y-1)^{2}\right)\right],
\end{aligned}
$$

taken to induce nontrivial dynamical structures. We observed in Figure 3 that the solution is bounded for $t>0$, and, at smaller time, say $t=0.1$, two separate structures interpreted as isolation of the species population emerged (like solitons), but as simulation time is increasing, the two separate structures combined as one. At $t>6$, the domain is completely covered by the species which shows that the population of $u$ is increasing with time. This result guarantees the permanence and existence of model (34).

For the second case, we consider the initial condition

$$
\begin{gathered}
u(x, y, 0)=11.3 \exp \left(-10\left((x+2)^{2}+(y+4)^{2}\right)\right), \\
+10 \exp \left(-10\left((x-2)^{2}+(y-2)^{2}\right)\right), \\
+10.2 \exp \left(-15\left((x+2)^{2}+(y-4)^{2}\right)\right),
\end{gathered}
$$

to mimic a case with four peaks.

In Figure 4, we present the numerical solution of problem (34) with initial population (36). To ensure the long-term survival of the species, we increase the growth rate $\tau$. In the simulations, at $t=0.1$ we observe the emergence of four peaks which may represent the existence of species in a confined area of dimensions: $x=y$. As simulation time 


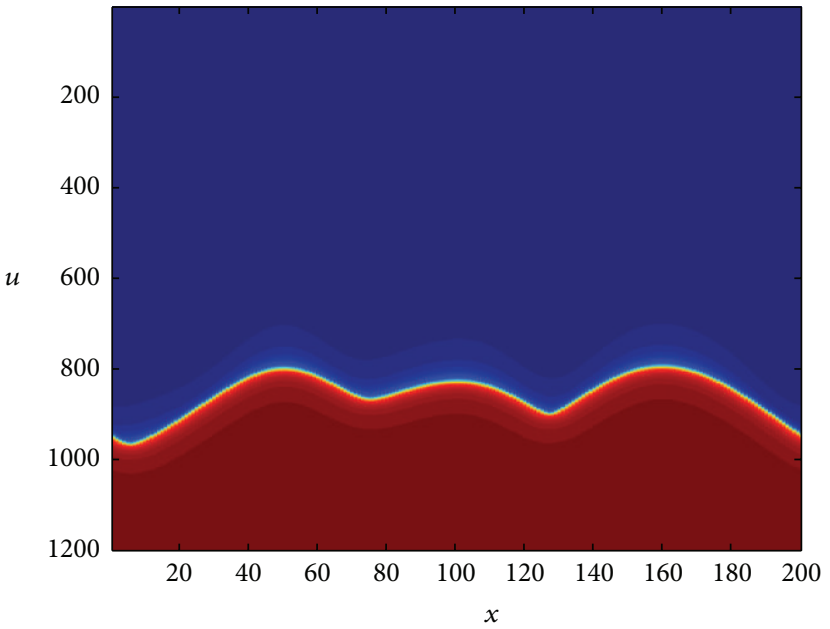

(a)

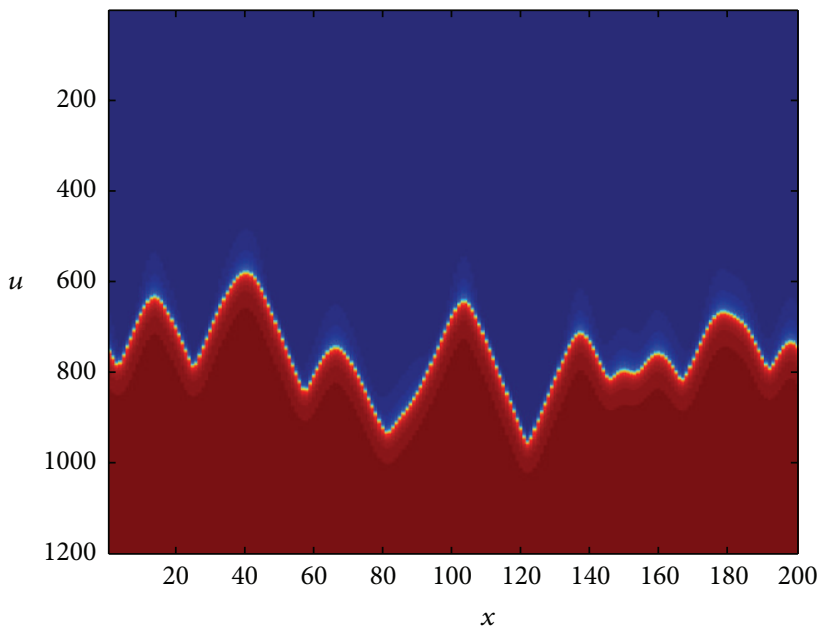

(c)

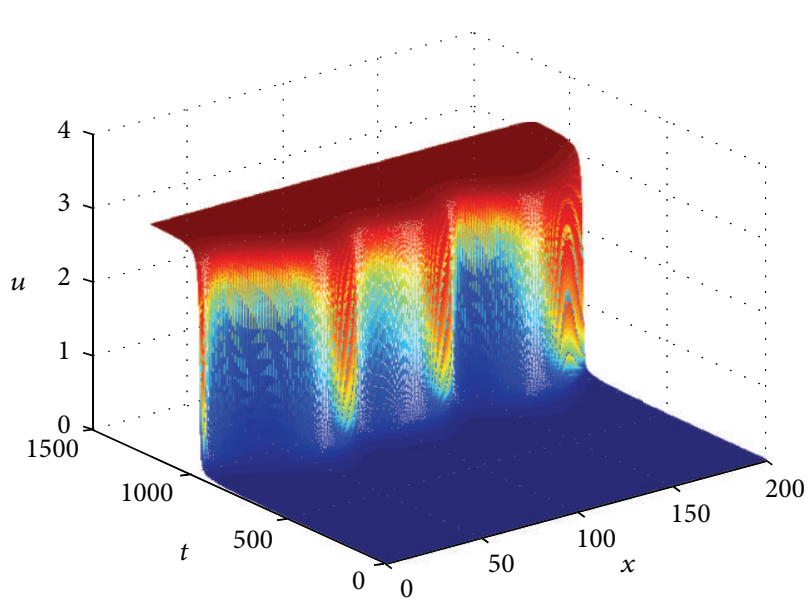

(b)

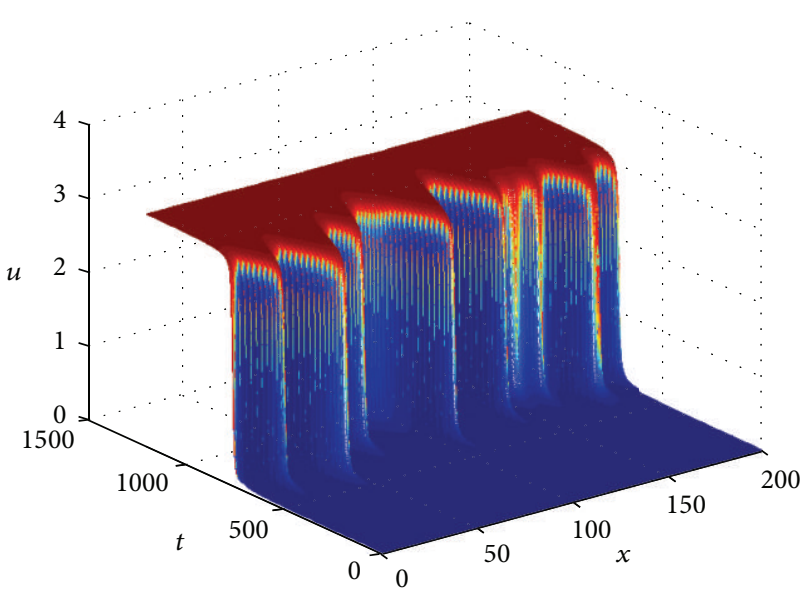

(d)

Figure 2: The snapshots (a, c) and surface plots (b, d) of one-dimensional KiSS model (32) showing the effect of diffusion (a) at $\delta=1$ and (b) at $\delta=0.1$. Simulation runs for $N=200$.
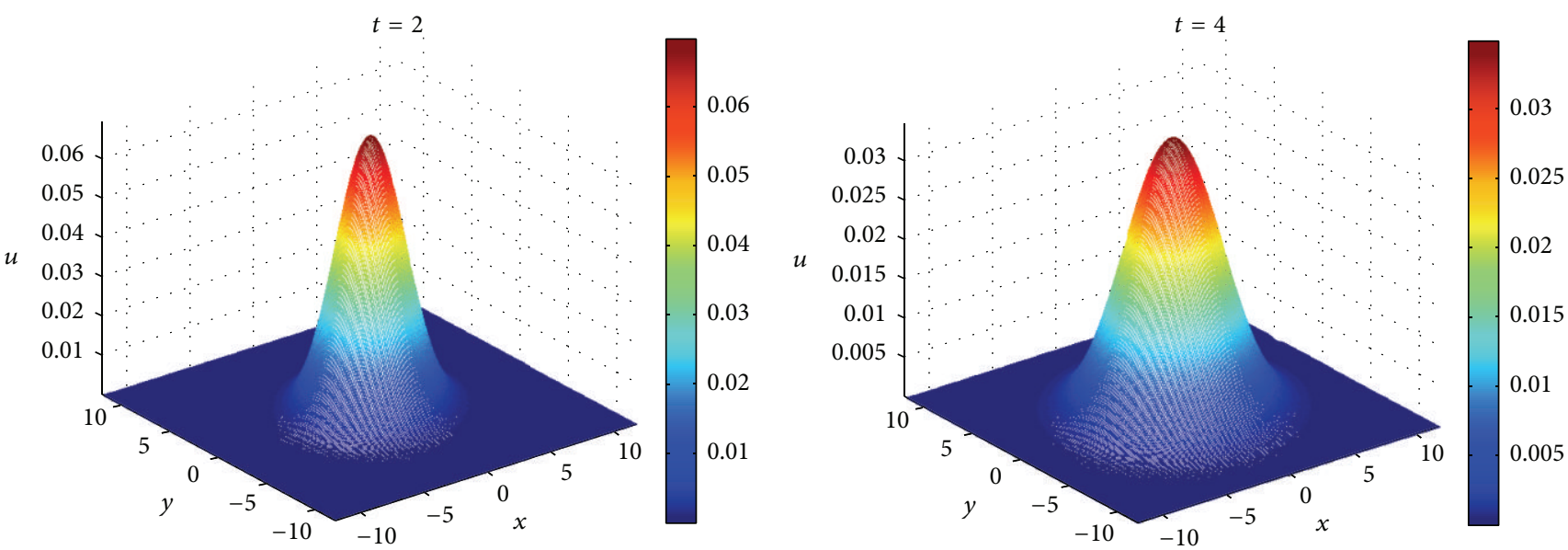

FIgURE 3: Persistence and existence of two-dimensional KiSS model (34) with initial population (35) at different time $t$ levels. Parameters are $\delta=0.5, p=2, \tau=2.5$, and $L=12$. 

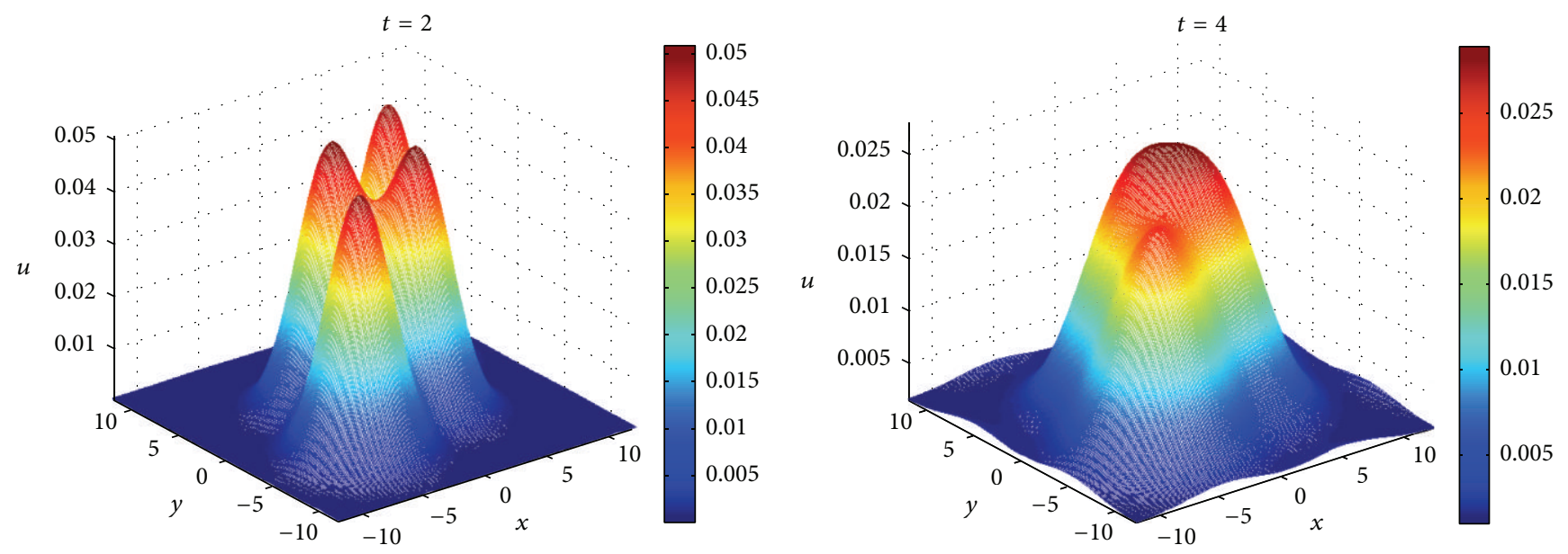

FIGURE 4: Persistence and existence of two-dimensional KiSS model (34) with initial population (36) at different time $t$ levels. Parameters are $\delta=0.5, p=2, \tau=3$, and $L=12$.

is increasing, so also the isolated species (representing four peaks) spread within the domain to become one. Readers are to compare the results obtained at $t=4$ in Figures 3 and 4 for clarity.

\section{Conclusion}

In this research paper, we have given an extension to the study of nonlinear reaction-diffusion problem. The case study is that of Kierstead, Slobodkin, and Skellam (KiSS) model, for population growing on a patch of finite size $L$. We investigate the model for permanence and existence of the species population over a period of time. Also, our aim has been to formulate and provide good working, versatile spectral methods in conjunction with the exponential time-differencing scheme, which avoids stiffness problem associated with the linear term of the reaction-diffusion problem. Numerical simulations of the diffusive KiSS model are provided to demonstrate and compare the theoretical results. It should be noted that the mathematical approach reported here can be extended to multispecies reaction-diffusion problems.

\section{Conflict of Interests}

The authors declare that there is no conflict of interests regarding the publication of this paper.

\section{Acknowledgment}

The research contained in this paper is supported by the South African National Research Foundation.

\section{References}

[1] H. Kierstead and L. B. Slobodkin, "The size of water masses containing plankton looms," Journal of Marine Research, vol. 12, pp. 141-147, 1953.

[2] J. G. Skellam, "Random dispersal in theoretical populations," Biometrika, vol. 38, pp. 196-218, 1951.
[3] A. Okubo, "Horizontal dispersion and critical scales for phytoplankton patches," in Spatial Pattern in Plankton Communities, vol. 3 of NATO Conference Series, pp. 21-42, Springer, New York, NY, USA, 1978.

[4] V. Méndez, S. Fedotov, and W. Horsthemke, Reaction-Transport Systems: Mesoscopic Foundations, Fronts, and Spatial Instabilities, Springer, Berlin, Germany, 2010.

[5] J. D. Murray, Mathematical Biology I: An Introduction, Springer, New York, NY, USA, 2002.

[6] K. Ishige and H. Yagisita, "Blow-up problems for a semilinear heat equations with large diffusion," Journal of Differential Equations, vol. 212, pp. 114-128, 2005.

[7] A. A. Lacey, "Diffusion models with blow-up," Journal of Computational and Applied Mathematics, vol. 97, no. 1-2, pp. 3949, 1998.

[8] T. Małolepszy and W. Okrasiñski, "Blow-up time for solutions to some nonlinear Volterra integral equations," Journal of Mathematical Analysis and Applications, vol. 366, no. 1, pp. 372384, 2010.

[9] K. M. Owolabi, "Robust numerical solution of the time-dependent problems with blow-up," International Journal of Bioinformatics and Biomedical Engineering, vol. 1, no. 2, pp. 53-63, 2015.

[10] F. B. Weissler, "Single point blow-up for a semilinear initial value problem," Journal of Differential Equations, vol. 55, no. 2, pp. 204-224, 1984.

[11] B. Fornberg, A Practical Guide to Pseudospectral Methods, Cambridge University Press, Cambridge, UK, 1998.

[12] J. P. Boyd, Chebyshev and Fourier Spectral Methods, Dover, New York, NY, USA, 2001.

[13] L. N. Trefethen, Spectral Methods in MATLAB, SIAM, Philadelphia, Pa, USA, 2000.

[14] J. A. C. Weideman and S. C. Reddy, "A MATLAB differenciation suite," Transactions on Mathematical Software, vol. 26, no. 4, pp. 465-519, 2001.

[15] B. Fornberg and T. A. Driscoll, "A fast spectral algorithm for nonlinear wave equations with linear dispersion," Journal of Computational Physics, vol. 155, no. 2, pp. 456-467, 1999.

[16] S. M. Cox and P. C. Matthews, "Exponential time differencing for stiff systems," Journal of Computational Physics, vol. 176, no. 2, pp. 430-455, 2002. 
[17] Q. Du and W. Zhu, "Analysis and applications of the exponential time differencing schemes and their contour integration modifications," BIT Numerical Mathematics, vol. 45, no. 2, pp. 307328, 2005.

[18] A.-K. Kassam and L. N. Trefethen, "Fourth-order time-stepping for stiff PDEs," SIAM Journal on Scientific Computing, vol. 26, no. 4, pp. 1214-1233, 2005.

[19] K. M. Owolabi and K. C. Patidar, "Higher-order time-stepping methods for time-dependent reaction-diffusion equations arising in biology," Applied Mathematics and Computation, vol. 240, pp. 30-50, 2014.

[20] S. Krogstad, "Generalized integrating factor methods for stiff PDEs," Journal of Computational Physics, vol. 203, no. 1, pp. 7288, 2005.

[21] H. Munthe-Kaas, "High order Runge-Kutta methods on manifolds," Applied Numerical Mathematics, vol. 29, pp. 115-127, 1999.

[22] K. M. Owolabi and K. C. Patidar, "Numerical solution of singular patterns in one-dimensional Gray-Scott-like models," International Journal of Nonlinear Sciences and Numerical Simulation, vol. 15, no. 7-8, pp. 437-462, 2014. 


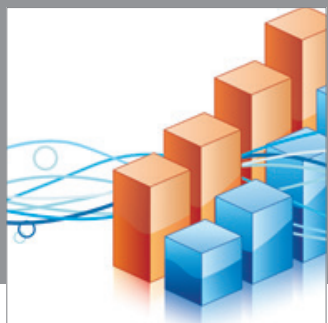

Advances in

Operations Research

mansans

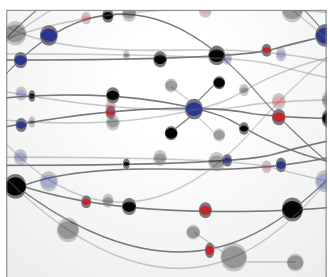

The Scientific World Journal
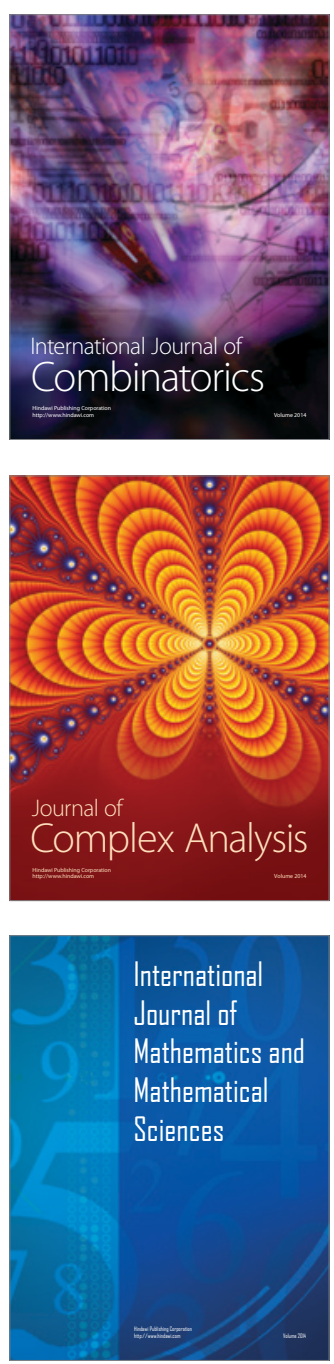
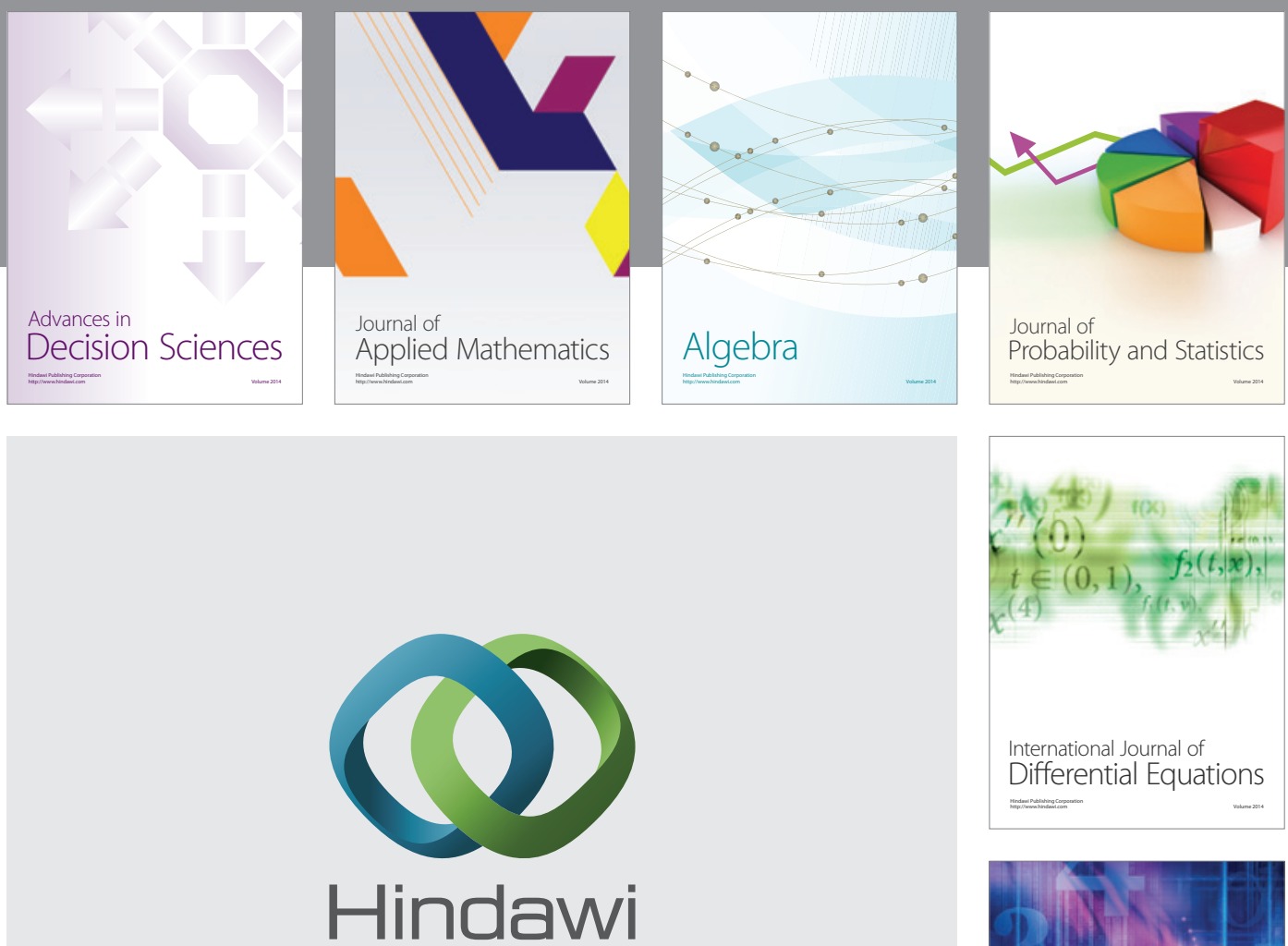

Submit your manuscripts at http://www.hindawi.com
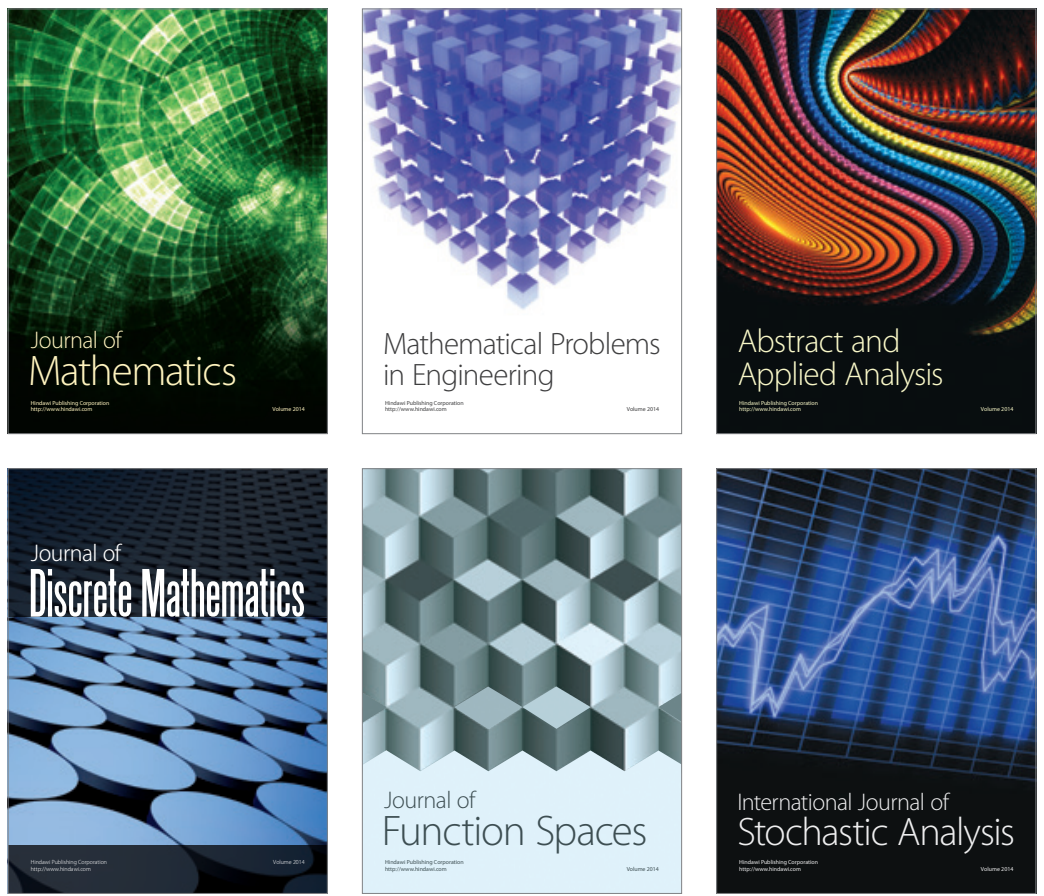

Journal of

Function Spaces

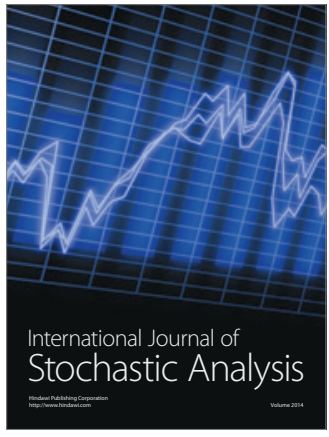

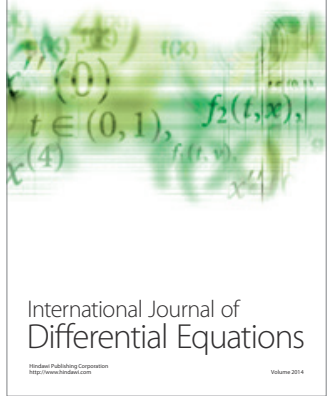
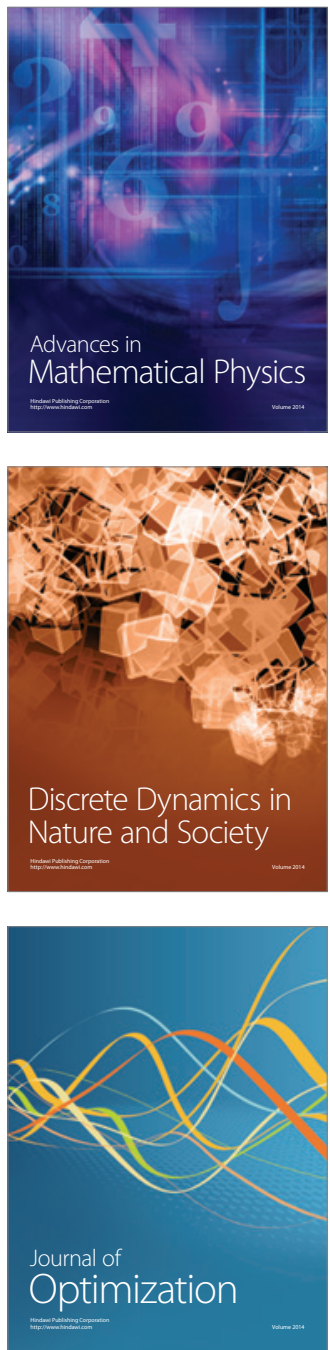